\title{
Interaction between silica and hydrophobic cations
}

\author{
J. DEPASSE
}

From the Université Libre de Bruxelles, Chimie Générale I, 50, av. F. Roosevelt, 1050 Brussels, Belgium

ABSTRACT The interactions between silica and some molecules which have a high affinity for its surface have been studied. The hydrophobic properties and the positive charge of these molecules are likely to be responsible for their strong adsorption on to silica. These observations should be useful in research into new inhibitors of the effects of silica. One of the cations tested, chloroquine, has been shown to be an effective inhibitor of the haemolytic activity of quartz.

Silica particles are negatively charged, and cations can be adsorbed on to their surface. The interaction between silica and some large hydrophobic cations has been investigated in order to find molecules with a high affinity for silica. Coupling of macromolecules or proteins with such molecules could increase their affinity for the surface of silica, and provide new inhibitors of silicosis.

The interaction between silica and cations is easily studied by means of coagulation experiments. Mixing a salt with a hydrosol leads to coagulation, a phenomenon that can be observed by increased optical density of the sol. For a sol composed of negatively charged particles, the coagulation concentration of the salt depends on the nature of the cation. A low coagulation concentration is related to a high affinity of the cation for the particle surface. Except for ammonium ions, the cations that have been tested in this study have a partially planar configuration, which should favour adsorption on silica. Of the compounds tested, chloroquine, quinacrine and quinine are anti-malarial drugs. Paraquat is highly toxic, and was tested merely for comparison.

The influence of chloroquine on the haemolytic activity of quartz has also been examined.

\section{Materials and methods}

\section{MATERIALS}

Silica

Ludox HS silica hydrosol was obtained from Dupont De Nemours. The spherical particles, composed of

Received for publication 14 July 1976 Accepted for publication 28 September 1976 amorphous silica, were approximately $15 \mathrm{~nm}$ in diameter. Quartz was obtained from Merck and was thoroughly washed with phosphoric acid. After grinding, the particles had diameters of $1-5 \mu \mathrm{m}$.

\section{Cations}

Paraquat (1,1'-dimethyl, 4,4'-dipyridilium chloride), was kindly provided by ICI. Chloroquine phosphate and quinine sulphate were purchased from the Pharmacie Centrale Belge, quinacrine hydrochloride from Federa, tetramethylammonium bromide (TMABr) from the Union Chimique Belge, and 1-cyclohexyl-3-(2-morpholinoethyl) carbodiimide metho-p-toluene sulphonate from the Aldrich Chemical Corporation, Inc.

\section{Sheep erythrocytes}

These were obtained from the Institut Pasteur, Brussels, and were washed at least four times with isotonic saline solution, before use.

\section{Methods}

\section{COAGULATION}

In each coagulation run, one set of test tubes was filled with solutions of appropriate salt concentrations. The $\mathrm{pH}$ was fixed at 7.5 by means of a phosphate buffer. The hydrosol was then rapidly added, and mixed using a vortex shaker. The final $\mathrm{SiO}_{2}$ concentration was $10 \mathrm{~g} / \mathrm{l}$. The optical density was read at $400 \mathrm{~nm}$, after incubation for one hour at $25^{\circ} \mathrm{C}$.

\section{ADSORPTION}

Silica sols $(30 \mathrm{~g} / \mathrm{l})$ were mixed with carbodiimide or 
with quinacrine $\left(1.25 \times 10^{-2} \mathrm{~mol} / \mathrm{l}\right)$. After equilibration, the silica was centrifuged off, and carbodiimide or quinacrine measured spectrophotometrically in the supernatant.

HAEMOLYSIS

Sheep erythrocytes $\left(10^{7}\right.$ cells $\left./ \mathrm{ml}\right)$ and quartz particles $\left(1.5 \times 10^{8}\right.$ particles $\left./ \mathrm{ml}\right)$ were incubated for one hour at $37^{\circ} \mathrm{C}$ in isotonic $\mathrm{NaCl}$-phosphate solu-

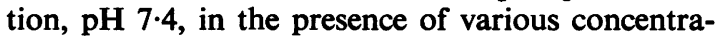
tions of chloroquine. The suspension was then centrifuged at $2000 \mathrm{~g}$ for five minutes, and the optical density of the supernatant was measured at $410 \mathrm{~nm}$ in order to determine the quantity of released haemoglobin and thus the degree of haemolysis.

\section{Results}

\section{COAGULATION}

The behaviour of $\mathrm{NH}_{4}{ }^{+}$has been described in a previous paper (Depasse and Warlus, 1976) and it is included in Fig. 1 for comparison. All the hydrophobic cations are much more effective than the ammonium ion in coagulating silica hydrosols. This Figure also shows the approximate coagulation concentration of quinacrine, estimated visually.

\section{ADSORPTION}

It was observed that $35 \%$ of the quinacrine was adsorbed on the particles whereas, under our experimental conditions, the proportion of adsorbed carbodiimide was too small to be measured. This confirms that a low coagulation concentration is related to a strong affinity of the cation for the surface of silica.

\section{HAEMOLYSIS}

Figure 2 shows that chloroquine very effectively inhibits haemolysis by quartz. Chloroquine is probably the first non-polymeric molecule which has been shown to inhibit the haemolytic activity of quartz, at low concentrations.

\section{Discussion}

It has been shown previously that the high affinity of $\mathrm{TMA}^{+}$(coagulation concentration around $10^{-2}$ $\mathrm{mol} / \mathrm{l}$ ) and the low affinity of $\mathrm{NH}_{4}+$ for the surface of silica is likely to be responsible for the toxicity of silica to membranes (Depasse and Warlus, 1976) and for the weak inhibitory action of lysozyme against the haemolytic activity of silica (Depasse and Léonis, 1976). TMA $^{+}$is more hydrophobic than $\mathrm{NH}_{4}{ }^{+}$. The hydrophobic cations that have been tested in this study possess aliphatic and/or aromatic groups, and they are probably even more hydro-

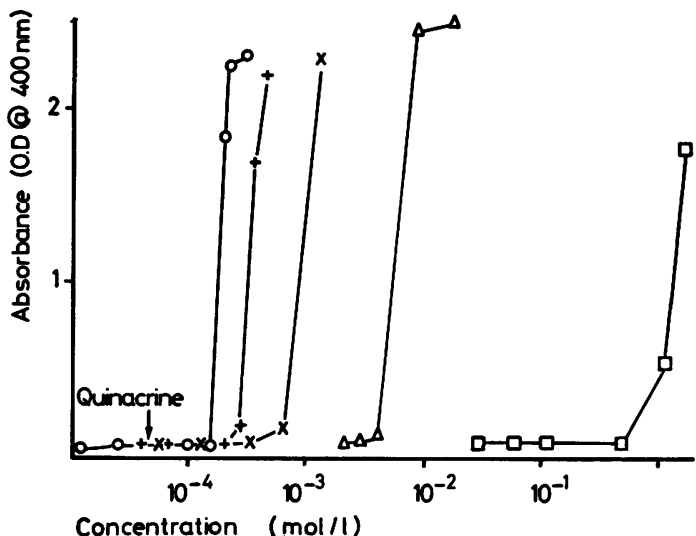

Fig. 1 Absorbance of $\mathrm{SiO}_{2}$ hydrosols, after incubation for $1 \boldsymbol{h}$ with various concentrations of chloroquine $(O)$, paraquat $(+)$, quinine $(\times)$, carbodiimide $(\triangle)$, or $\mathrm{NH}_{4}{ }^{+}$ ( $\square)$. The coagulation concentration of quinacrine is also indicated.

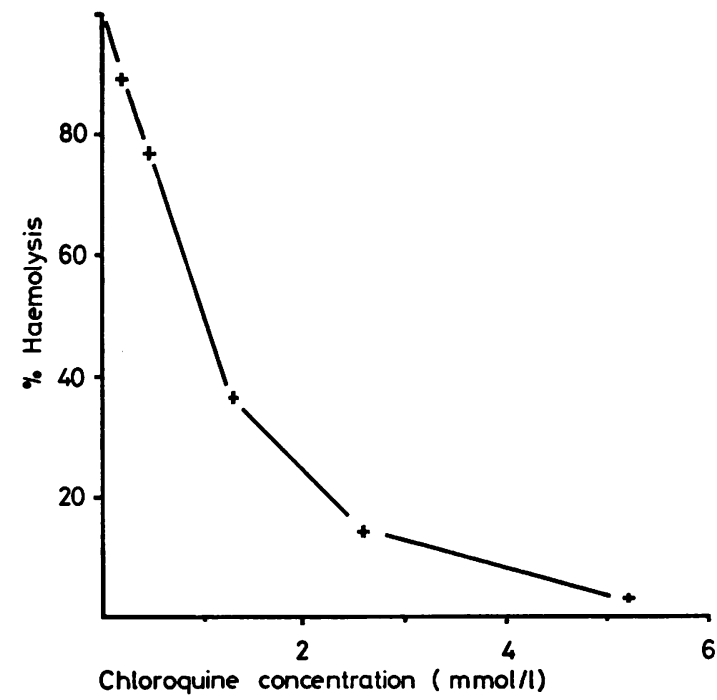

Fig. 2 Percentage haemolysis of sheep erythrocytes by quartz, as a function of the concentration of chloroquine.

phobic than TMA + . Accordingly, they have a greater affinity than $\mathrm{TMA}^{+}$for silica. Their coagulation concentrations are from two to four orders of magnitude lower than that of $\mathrm{NH}_{4}{ }^{+}$.

Quinacrine, chloroquine and paraquat are mainly bivalent at $\mathrm{pH} 7 \cdot 4$, and they are more effective than quinine and carbodiimide which are monovalent.

Comparison between quinacrine and chloroquine shows that substitution of the quinoleine group by 
the acridine group, which confers increased hydrophobic properties, also increases the affinity for silica.

Paraquat is the sole aromatic cation tested here which does not possess lone pair electrons. The fact that paraquat is as effective as the other cations for the coagulation of silica indicates that the hydrogen acceptor properties of those cations is of no importance for their interaction with silica. It should be noted that the adsorption of polyvinylpyridine$\mathrm{N}$-oxide and other polymers on silica (Holt, 1971) is generally attributed to the formation of hydrogen bonds between the polymer and silica (Nash et al., 1966). Those polymers are basic and generally possess aromatic groups, often derived from the pyridine ring. The present study suggests that it is not the hydrogen bond interaction but the hydrophobic and electrostatic interactions between the polymer and silica which are responsible for the adsorption.

In view of its anti-inflammatory action, chloroquine has been tested by Beck and Schlipkoter (1960) for a possible effect upon silicosis. In spite of the fact that chloroquine becomes concentrated in lysosomes (De Duve et al., 1974), where silica is thought to exert its toxic action (Allison et al., 1966), it has no influence on experimental silicosis. However, coupling of molecules which have a strong affinity for silica, with macromolecules or proteins, could increase their efficiency. This opinion is sup- ported by the fact that coupling of carbodiimide with lysozyme drastically increases the inhibitory activity of the protein against haemolysis by quartz (Depasse and Léonis, 1976).

I should like to thank Professor J. Léonis for his support. Financial support from the CEN-ULB is gratefully acknowledged.

References

Allison, A. C., Harington, J. S., and Birbeck, M. (1966). An examination of the cytotoxic effect of silica on macrophages. Journal of Experimental Medicine, 124, 141-154.

Beck, E. G., and Schlipkoter, H. W. (1960). Die Wirkung von Prethcamide, Chloroquine und Dinitrophenol auf die experimentelle Silikose bei weisen Ratten. Medizinische Welt, 49, 2596-2599.

De Duve, C., De Barsy, T., Poole, B., Trouet, A., Tulkens, P., and Van Hoof, F. (1974). Lysosomotropic agents. Biochemical Pharmacology, 23, 2495-2531.

Depasse, J., and Léonis, J. (1976). Inhibition of the hemolytic activity of quartz, by a chemically modified lysozyme. Environmental Research, 12, 371-374.

Depasse, J., and Warlus, J. (1976). Relation between the toxicity of silica and its affinity for tetraalkylammonium groups. Journal of Colloid Interface Science, 56, 618-621.

Holt, P. F. (1971). Poly(vinylpyridine oxides) in pneumoconiosis research. British Journal of Industrial Medicine, 28, 72-77.

Nash, T., Allison, A. C., and Harington, J. S. (1966). Physicochemical properties of silica in relation to its toxicity. Nature, 210, 259-261. 